\title{
THE CONTACT OF A CUBIC SURFACE WITH AN ANALYTIC SURFACE*
}

\author{
BY \\ ERNEST P. LANE

\section{INTRODUCTION}

The projective differential geometry of a surface in the neighborhood of one of its points has been enriched by the consideration of various quadrics covariant to the surface. Among these are the quadrics of Darboux, the quadric of Lie, and the canonical quadric of Wilczynski. All of these are members of the three-parameter family of quadrics having contact of the second order with the surface at the point considered. There is no nonsingular quadric having contact of the third order at an ordinary point of an unrestricted surface. In fact, if there exists a non-singular quadric having contact of the third order, at a general point of a surface, then the surface itself is a quadric, and there is a pencil of quadrics having contact of the third order at each point. All of these pencils contain, of course, the surface itself.

It is the purpose of this paper to investigate the contact of a cubic surface with an analytic surface and to determine necessary and sufficient conditions that a surface be a cubic. Since a cubic is determined by nineteen points, and since it is necessary to impose $(n+1)(n+2) / 2$ conditions to make an algebraic surface have contact of order $n$ with an analytic surface, it follows that there is a four-parameter family of cubics having contact of order four at a point of an analytic surface. There is no non-composite cubic with contact of order five at a general point of a surface, unless the surface is restricted to be itself a cubic. There is a pencil of cubics with contact of the fifth order at each point of a cubic surface. These remarks suffice to indicate the trend of the following investigation.

\section{Power Series EXPansions}

Let the four homogeneous coördinates $y^{(1)}, \cdots, y^{(4)}$ of a general point $y$ on a non-degenerate non-ruled surface $S$ be given as analytic functions of two independent variables $u, v$. If the curves $u=$ const. and $v=$ const. are the asymptotics, then the four functions $y$ are solutions of a system of dif-

\footnotetext{
* Presented to the Society, September 9, 1926; received by the editors in July, 1926.
} 
ferential equations which can be reduced to Wilczynski's canonical form,

$$
y_{u u}+2 b y_{v}+f y=0, \quad y_{v v}+2 a^{\prime} y_{u}+g y=0,
$$

whose coefficients are functions of $u, v$ satisfying the following conditions of complete integrability:

$$
\begin{gathered}
a_{u u}^{\prime}+g_{u}+2 b a_{v}^{\prime}+4 a^{\prime} b_{v}=0, \\
b_{v v}+f_{v}+2 a^{\prime} b_{u}+4 b a_{u}^{\prime}=0, \\
g_{u u}+4 g b_{v}+2 b g_{v}=f_{v v}+4 f a_{u}^{\prime}+2 a^{\prime} f_{u} .
\end{gathered}
$$

The derivatives of $y$ of all orders can be expressed uniquely as linear combinations of $y, y_{u}, y_{v}, y_{u v}$. We shall need the derivatives up to those of the fifth order inclusive, but we shall not need the coefficient of $y$ for the derivatives of the fourth order, and we shall need only the coefficient of $y_{w v}$ for the fifth order. The needed formulas will now be written, with the accent omitted from the $a^{\prime}$, for purposes of symmetry and simplicity:

$$
\begin{aligned}
y_{u u u} & =-f_{u} y-f y_{u}-2 b_{u} y_{v}-2 b y_{u v}, \\
y_{u u v} & =\left(2 b g-f_{v}\right) y+4 a b y_{u}-\left(f+2 b_{v}\right) y_{v}+0, \\
y_{u v v} & =\left(2 a f y-g_{u}\right) y-\left(g+2 a_{u}\right) y_{u}+4 a b y_{v}+0, \\
y_{v v v} & =-g_{v} y-2 a_{v} y_{u}-g y_{v}-2 a y_{u v} ; \\
y_{u u u u} & =\cdots-2\left(f_{u}+4 a b^{2}\right) y_{u}+2\left(2 b f+2 b b_{v}-b_{u u}\right) y_{v}-4 b_{u} y_{u v}, \\
y_{u u u v} & =\cdots+\left(4 a_{u} b+4 a b_{u}+2 b g-f_{v}\right) y_{u}-\left(f_{u}+2 b_{u v}+8 a b^{2}\right) y_{v}, \\
& -\left(f+2 b_{v}\right) y_{u v}, \\
(3) \quad y_{u u v v}= & +2\left(a f+4 a b_{v}+2 a_{v} b\right) y_{u}+2\left(b g+4 a_{u} b+2 a b_{u}\right) y_{v}+4 a b y_{u v}, \\
y_{u v v v}= & -\left(g+2 a_{u v}+8 a^{2} b\right) y_{u}+\left(4 a_{v} b+4 a b_{v}+2 a f-g_{u v}\right) y_{v}, \\
y_{v v v v}= & \cdots+2\left(2 a g+2 a_{u}-a_{v v}\right) y_{u}-2\left(g_{v}+4 a^{2} b\right) y_{v}-4 a_{v} y_{u v} ; \\
y_{u u u u v}= & \cdots+2\left(2 b f+2 b b_{v}-3 b_{u u}\right) y_{u v}, \\
y_{u u u v v}= & \cdots-2\left(f_{u}+4 a b^{2}+2 b_{u v}\right) y_{u v}, \\
y_{u u u v v} & =\cdots+2\left(b g+6 a_{u} b+4 a b_{u}\right) y_{u v}, \\
y_{u u v v v} & =\cdots+2\left(a f+6 a b_{v}+4 a_{v} b\right) y_{u v}, \\
y_{u v v v v} & =\cdots-2\left(g_{v}+4 a^{2} b+2 a_{u v}\right) y_{u v}, \\
y_{v v v v v} & =\cdots+2\left(2 a g+2 a a_{u}-3 a_{v v}\right) y_{u v} .
\end{aligned}
$$

If the points $y, y_{u}, y_{v}, y_{u v}$ are used as the vertices of a local tetrahedron of reference, with unit point suitably chosen, then the coördinates of a point 
$x$ on $S$ near the point $y$ may be easily calculated as power series in the increments $\Delta u$ and $\Delta v$ corresponding to displacement from $P_{y}$ to $P_{z}$ on $S$. We shall now write these series to as many terms as needed, using $\epsilon=\Delta u, \eta=\Delta v$ :

$$
\begin{aligned}
x_{1}=1 & -\frac{1}{2}\left(f \epsilon^{2}+g \eta^{2}\right)-\frac{1}{6} f_{u} \epsilon^{3}+\frac{1}{2}\left(2 b g-f_{v}\right) \epsilon^{2} \eta \\
& +\frac{1}{2}\left(2 a f-g_{u}\right) \epsilon \eta^{2}-\frac{1}{6} g_{v} \eta^{3}+\cdots, \\
x_{2}= & \epsilon-a \eta^{2}-\frac{1}{6} f \epsilon^{3}+2 a b \epsilon^{2} \eta-\frac{1}{2}\left(g+2 a_{u}\right) \epsilon \eta^{2}-\frac{1}{3} a_{v} \eta^{3} \\
& -\frac{1}{12}\left(f_{u}+4 a b^{2}\right) \epsilon^{4}+\frac{1}{6}\left(4 a_{u} b+4 a b_{u}+2 b g-f_{v}\right) \epsilon^{3} \eta+\frac{1}{2}\left(a f+4 a b_{v}\right. \\
& \left.+2 a_{v} b\right) \epsilon^{2} \eta^{2}-\frac{1}{6}\left(g_{v}+2 a_{u v}+8 a^{2} b\right) \epsilon \eta^{3} \\
& +\frac{1}{6}\left(a g+a a_{u}-\frac{1}{2} a_{v v}\right) \eta^{4}+\cdots,
\end{aligned}
$$

(4) $x_{2}=\eta-b \epsilon^{2}-\frac{1}{3} b_{u} \epsilon^{3}-\frac{1}{2}\left(f+2 b_{v}\right) \epsilon^{2} \eta+2 a b \epsilon \eta^{2}-\frac{1}{6} g \eta^{3}$

$$
\begin{aligned}
+ & \frac{1}{6}\left(b f+b b_{v}-\frac{1}{2} b_{u v}\right) \epsilon^{4}-\frac{1}{6}\left(f_{u}+2 b_{u v}+8 a b^{2}\right) \epsilon^{3} \eta \\
+ & \frac{1}{2}\left(b g+4 a_{v} b+2 a b_{v}\right) \epsilon^{2} \eta^{2}+\frac{1}{6}\left(4 a_{v} b+4 a b_{v}+2 a f-g_{u}\right) \eta^{3} \\
& -\frac{1}{12}\left(g_{v}+4 a^{2} b\right) \eta^{4}+\cdots, \\
x_{4}= & \epsilon \eta-\frac{1}{3}\left(b \epsilon^{3}+a \eta^{3}\right)-\frac{1}{6} b_{u} \epsilon^{4}-\frac{1}{6}\left(f+2 b_{v}\right) \epsilon^{2} \eta+a b \epsilon^{2} \eta^{2} \\
& -\frac{1}{6}\left(g+2 a_{u}\right) e \eta^{2}-\frac{1}{6} a_{v} \eta^{4}+\frac{1}{60}\left(2 b f+2 b b_{v}-3 b_{u v}\right) \epsilon^{5} \\
& -\frac{1}{12}\left(f_{v}+2 b_{u v}-4 a b^{2}\right) \epsilon^{4} \eta+\frac{1}{6}\left(b g+6 a_{v} b+4 a b_{u}\right) \epsilon^{2} \eta^{2} \\
& +\frac{1}{6}\left(a f+4 a_{v} b+6 a b_{v}\right) \epsilon^{2} \eta^{3}-\frac{1}{12}\left(g v+2 a_{u v}+4 a^{2} b\right) \epsilon \eta^{4} \\
+ & \frac{1}{60}\left(2 a g+2 a a_{v}-3 a_{v v}\right) \eta^{5}+\cdots
\end{aligned}
$$




\section{Cubics with various contacts}

Let us write the equation of a cubic surface in the form

$$
a_{111} x_{1}^{3}+\cdots+a_{284} x_{2} x_{8} x_{4}=0,
$$

the subscripts in the case of each coefficient indicating the term to which it belongs. This cubic passes through the point $y$ if, and only if, $a_{111}=0$. It has first order contact with the surface $S$ at the point $y$ if also $a_{112}=a_{113}=0$. It has second order contact if also $a_{122}=a_{133}=a_{114}+a_{123}=0$, as may be verified by observing that these conditions are necessary and sufficient that the power series for the twenty possible cubic combinations of the $x_{i}$ as obtained from (4) satisfy equation (5) identically in $\epsilon$ and $\eta$ up to, and including, terms of the second order. Similarly, conditions necessary and sufficient for third order contact are found to be, in addition to those just mentioned,

$$
a_{222}=\frac{2}{3} b a_{123}, \quad a_{333}=\frac{2}{3} a a_{123}, a_{124}+a_{223}=0, a_{134}+a_{233}=0 .
$$

The conditions for fourth order contact may be reduced to

$$
\begin{aligned}
& a_{228}=-\frac{1}{4} \frac{b_{u}}{b} a_{128}, a_{283}=-\frac{1}{4} \frac{a_{v}}{a} a_{123}, a_{284}+a_{144}=0, \\
& a_{224}=\left(\frac{2}{3} b_{v}-\frac{1}{6} b \frac{a_{v}}{a}\right) a_{123}, a_{334}=\left(\frac{2}{3} a_{u}-\frac{1}{6} a \frac{b_{u}}{b}\right) a_{123},
\end{aligned}
$$

in addition to the foregoing, so that the equation of the most general cubic having contact of the fourth order with the surface $S$ at the point $y$ may be written in the form

$$
\begin{aligned}
& a_{123}\left[\frac{2}{3}\left(b x_{2}^{8}+a x_{3}^{8}\right)+\left(x_{2} x_{8}-x_{1} x_{4}\right)\left(x_{1}-\frac{1}{4} \frac{b_{u}}{b} x_{2}-\frac{1}{4} \frac{a_{v}}{a} x_{2}\right)\right. \\
& \left.\quad+\left(\frac{2}{3} b_{v}-\frac{1}{6} b \frac{a_{v}}{a}\right) x_{2}^{2} x_{4}+\left(\frac{2}{3} a_{u}-\frac{1}{6} a \frac{b_{u}}{b}\right) x_{3}^{2} x_{4}\right] \\
& \quad+a_{144}\left(x_{1} x_{4}-x_{2} x_{3}\right) x_{4}+a_{244} x_{2} x_{4}^{2}+a_{344} x_{3} x_{4}^{2}+a_{444} x_{4}^{3}=0 .
\end{aligned}
$$

When the terms of the fifth order are substituted from the power series for the cubic combinations of the $x_{i}$ into equation (6), the result may be arranged in the form 


$$
\begin{aligned}
& \left\{\frac{2}{15} b a_{123}\left[f-\frac{2}{3} b_{v}-\frac{5}{6} b \frac{a_{v}}{a}-\frac{1}{4} \frac{b_{u u}}{b}+\frac{5}{16}\left(\frac{b_{u}}{b}\right)^{2}\right]\right\} \epsilon^{5} \\
+ & \left\{\frac{2}{3} b a_{144}-\frac{1}{6} b a_{123}\left[\frac{b_{u v}}{b}-\frac{b_{u} b_{v}}{b^{2}}-16 a b-\frac{1}{4} \frac{a_{v}}{a} \frac{b_{u}}{b}\right]\right\} \epsilon^{4} \eta \\
+ & \left\{a_{244}-\frac{2}{3} b a_{123}\left[g+\frac{7}{6} a \frac{b_{u}}{b}+\frac{10}{3} a_{u}+\frac{1}{2} \frac{b_{v v}}{b}-\frac{1}{4} \frac{a_{v}}{a} \frac{b_{v}}{b}\right]\right\} \epsilon^{3} \eta^{2} \\
+ & \cdots=0,
\end{aligned}
$$

the omitted terms being obtained from those written by interchange of $a$ and $b, f$ and $g, u$ and $v, 2$ and $3, \epsilon$ and $\eta$. If this equation is satisfied identically in $\epsilon$ and $\eta$ without restriction on the surface $S$, the cubic (6) reduces to $x_{1}^{8}=0$, that is, the tangent plane counted three times. If the cubic (6) is restricted to be non-composite, then $a_{123} \neq 0$, and equation (7) will be satisfied identically in $\epsilon$ and $\eta$ provided that the surface $S$ is restricted by the three conditions

$$
\begin{gathered}
\frac{\partial^{2}}{\partial u \partial v} \log \frac{a}{b}=0 \\
f=\frac{2}{3} b_{v}+\frac{5}{6} b \frac{a_{v}}{a}+\frac{1}{4} \frac{b_{u u}}{b}-\frac{5}{16}\left(\frac{b_{u}}{b}\right)^{2} \\
g=\frac{2}{3} a_{u}+\frac{5}{6} a \frac{b_{u}}{b}+\frac{1}{4} \frac{a_{v v}}{a}-\frac{5}{16}\left(\frac{a_{v}}{a}\right)^{2}
\end{gathered}
$$

and provided that the coefficients of the cubic (6) satisfy three conditions which can easily be written down. Then all the cubics represented by the following equation have contact of the fifth order with the surface $S$ :

$$
\begin{aligned}
& \frac{2}{3}\left(b x_{2}^{8}+a x_{3}^{8}\right)+\left(x_{2} x_{3}-x_{1} x_{4}\right)\left(x_{1}-\frac{1}{4} \frac{b_{u}}{b} x_{2}-\frac{1}{4} \frac{a_{v}}{a} x_{3}\right. \\
- & \left.\frac{1}{4}\left[\frac{\partial}{\partial v \partial u} \log b-16 a b-\frac{1}{4} \frac{a_{v}}{a} \frac{b_{u}}{b}\right] x_{4}\right) \\
+ & \left(\frac{2}{3} b_{v}-\frac{1}{6} b \frac{a_{v}}{a}\right) x_{2}^{2} x_{4}+\left(\frac{2}{3} a_{u}-\frac{1}{6} a \frac{b_{u}}{b}\right) x_{3}^{2} x_{4} \\
+ & \frac{2}{3} b\left(g+\frac{7}{6} a \frac{b_{u}}{b}+\frac{10}{3} a_{u}+\frac{1}{2} \frac{b_{v v}}{b}-\frac{1}{4} \frac{a_{v}}{a} \frac{b_{v}}{b}\right) x_{4}^{2} x_{2} \\
+ & \frac{2}{3} a\left(f+\frac{7}{6} b \frac{a_{v}}{a}+\frac{10}{3} b_{v}+\frac{1}{2} \frac{a_{u u}}{a}-\frac{1}{4} \frac{a_{u}}{a} \frac{b_{u}}{b}\right) x_{4}^{2} x_{3}+\lambda x_{4}^{8}=0,
\end{aligned}
$$

where $\lambda$ is an arbitrary function of $u, v$. 
One of my students, Miss Selma Learman, in her master's thesis (Chicago, 1926) has obtained conditions (8) as necessary conditions that a surface be a cubic. The lengthy and tedious calculations involved in reaching them were performed by both of us independently and checked by comparison.

\section{SIMPLIFICATION OF CONDITIONS}

The first of conditions (8) expresses that the surface $S$ is of the kind called* by Fubini isothermally asymptotic (isotermo-asintotica). It is on such a surface that the directrix curves of Wilczynski form a conjugate net. Since this condition implies that the ratio of $a$ to $b$ is a function of $u$ alone times a function of $v$ alone, it follows that it is possible, by means of a transformation which does not disturb the canonical form of equations (1), to reduce this ratio to unity so that we shall have $a=b$. We shall hereafter suppose, unless indicated to the contrary, that this transformation has been made. Then the first of conditions (8) is satisfied identically, and the other two become

$$
\begin{aligned}
& f=\frac{3}{2} b_{v}+\frac{1}{4} \frac{b_{u u}}{b}-\frac{5}{16}\left(\frac{b_{u}}{b}\right)^{2} \\
& g=\frac{3}{2} b_{u}+\frac{1}{4} \frac{b_{v v}}{b}-\frac{5}{16}\left(\frac{b_{v}}{b}\right)^{2} .
\end{aligned}
$$

Equation (9) becomes, in consequence of these conditions,

$$
\begin{aligned}
& \frac{2}{3} b\left(x_{2}^{3}+x_{8}^{8}\right)+\left(x_{2} x_{3}-x_{1} x_{4}\right)\left(x_{1}-\frac{1}{4} \frac{b_{u}}{b} x_{2}-\frac{1}{4} \frac{b_{v}}{b} x_{3}\right. \\
& \left.-\frac{1}{4}\left[\frac{\partial^{2}}{\partial u \partial v} \log b-16 b^{2}-\frac{1}{4} \frac{b_{u} b_{v}}{b^{2}}\right] x_{4}\right) \\
& +\frac{1}{2} b_{v} x_{2}^{2} x_{4}+\frac{1}{2} b_{u} x_{3}^{2} x_{4}+\frac{2}{3} b\left[6 b_{u}+\frac{3}{4} \frac{b_{u u}}{b}-\frac{9}{16}\left(\frac{b_{v}}{b}\right)^{2}\right] x_{2} x_{2} \\
& +\frac{2}{3} b\left[6 b_{v}+\frac{3}{4} \frac{b_{v v}}{b}-\frac{9}{16}\left(\frac{b_{u}}{b}\right)^{2}\right] x_{4}^{2} x_{3}+\lambda x_{4}^{3}=0 .
\end{aligned}
$$

By means of the relation $a=b$ and conditions (10), it is possible to reduce the first two of the integrability conditions (2) to the form

- Fubini and Cech, Geometria Proiettiva Differenziale, Bologna, Zanichelli, 1926, vol. I, p. 115. 


$$
\begin{aligned}
& {\left[\frac{\partial^{2}}{\partial u \partial v} \log b+12 b^{2}\right]_{v}=\left[\frac{1}{4}\left(\frac{b_{v}}{b}\right)^{2}-10 b_{v}\right]_{u},} \\
& {\left[\frac{\partial^{2}}{\partial u \partial v} \log b+12 b^{2}\right]_{u}=\left[\frac{1}{4}\left(\frac{b_{u}}{b}\right)^{2}-10 b_{v}\right]_{v} .}
\end{aligned}
$$

Differentiating the first of these equations with respect to $u$ and the second with respect to $v$, and subtracting, we obtain, after some reductions, the symmetric relation

$$
2 b b_{u u u}-b_{u} b_{u u}=2 b b_{v v v}-b_{v} b_{v v} .
$$

Direct calculation now suffices to show that the third of integrability conditions (2) is a consequence of the other two. Therefore, a surface $S$ which, at each of of its points, admits a cubic surface with contact of order five, is an integrating surface of the system of differential equations

$$
\begin{aligned}
& y_{u v}+2 b y_{v}+\left[\frac{3}{2} b_{v}+\frac{1}{4} \frac{b_{u u}}{b}-\frac{5}{16}\left(\frac{b_{u}}{b}\right)^{2}\right] y=0, \\
& y_{v v}+2 b y_{u}+\left[\frac{3}{2} b_{u}+\frac{1}{4} \frac{b_{v v}}{b}-\frac{5}{16}\left(\frac{b_{v}}{b}\right)^{2}\right] y=0,
\end{aligned}
$$

in which $b$ is a solution of equations (12).

A particular solution of equations (12) is $b=$ const. Then system (14) becomes

$$
y_{u u}+2 b y_{v}=0, \quad y_{v v}+2 b y_{u}=0,
$$

and four particular solutions thereof are

$$
y^{(1)}=1, y^{(2)}=e^{-2 b(u+v)}, y^{(3)}=e^{-2 b\left(\omega u+\omega^{2} v\right)}, y^{(1)}=e^{2 b\left(\omega^{2} u+\omega v\right)},
$$

where $\omega$ is a complex cube root of unity. If $u$ and $v$ are eliminated from these equations we find that the algebraic equation of this integrating surface is

$$
y^{(2)} y^{(3)} y^{(4)}=\left(y^{(1)}\right)^{3},
$$

or, in non-homogeneous coördinates, $x y z=1$. This is the cubic surface which appears in the projective theory as the analogue of the sphere, in the sense that the projective lines of curvature on this surface are indeterminate, the projective normals all passing through a fixed point.

V. THE PENCIL OF CUBICS WITH CONTACT OF THE FIFTH ORDER

At each point of a surface $S$ defined by equations (14) subject to the conditions of integrability (12) there is a pencil of cubic surfaces with contact of the fifth order, represented by equation (11). Let us consider a particular 
cubic of this family. As the point $y$ varies over the surface $S$ this cubic has an envelope, part of which, certainly, is the surface $S$. We shall proceed to find the points of contact of the cubic with its envelope.

If $x_{1}, \cdots, x_{4}$ are the coördinates of a point $x$ referred to the local tetrahedron $y, y_{u}, y_{v}, y_{u v}$ of a surface $S$ at a point $y$ and if $\xi_{1}, \ldots, \xi_{4}$ are the coördinates of the same point $x$ referred to the corresponding local tetrahedron at a point on $S$ near $P_{y}$ corresponding to increments $\epsilon$ and $\eta$ of $u$ and $v$ respectively, then the equations of transformation between these two tetrahedrons are, in general,

$$
\begin{aligned}
& \xi_{1}=x_{1}+f \epsilon x_{2}+g \eta x_{3}+\left[\left(f_{v}-2 b g\right) \epsilon+\left(g_{u}-2 a f\right) \eta\right] x_{4}+\cdots, \\
& \xi_{2}=-\epsilon x_{1}+x_{2}+2 a \eta x_{3}+\left[-4 a b \epsilon+\left(g+2 a_{u}\right) \eta\right] x_{4}+\cdots, \\
& \xi_{3}=-\eta x_{1}+2 b \epsilon x_{2}+x_{3}+\left[\left(f+2 b_{v}\right) \epsilon-4 a b \eta\right] x_{4}+\cdots, \\
& \xi_{4}=0-\eta x_{2}-\epsilon x_{3}+x_{4}+\cdots .
\end{aligned}
$$

Making $a=b$ we may find for the fifteen cubic combinations of the coördinates which appear in equation (11) the formulas of transformation, corresponding to displacement along the asymptotic $v=$ const. If we write the equation of the cubic corresponding to (11) at a point on $S$ near $P_{y}$ but referred, by means of these formulas of transformation, to the local tetrahedron at $P_{y}$; if we then subtract from this equation the equation (11); if we divide the result by $\epsilon$ and then let $\epsilon$ approach zero, we find the equation of a surface which intersects the cubic (11) in the points at which it touches its envelope as $u$ alone varies. This equation has precisely the same fifteen cubic combinations of the coördinates which appear in equation (11). Moreover, thirteen of these have coefficients which are independent of $\lambda$ and are precisely the same as the corresponding coefficients of (11), except perhaps for a common factor. The two terms whose coefficients contain $\lambda$ are the terms in $x_{3} x_{4}{ }^{2}$ and $x_{4}{ }^{3}$. Indicating, therefore, the sum of the other thirteen terms of equation (11) by $\phi$, we may write the new equation which we have obtained in the form

$$
\frac{1}{4} \frac{b_{u}}{b} \phi+\left(A^{(u)}-3 \lambda\right) x_{3} x_{4}^{2}+\left(B^{(u)}+\lambda_{u}\right) x_{4}^{3}=0,
$$

where

$$
\begin{aligned}
A^{(u)}= & \frac{2}{3} \frac{\partial}{\partial u}\left\{b\left[f+\frac{9}{2} b_{v}+\frac{1}{2} \frac{b_{u u}}{b}-\frac{1}{4}\left(\frac{b_{u}}{b}\right)^{2}\right]\right\}+b_{u}\left(f+2 b_{v}\right) \\
& -\frac{1}{4} \frac{b_{v}}{b}\left(2 b g-f_{v}\right)+b^{2}\left(\frac{\partial^{2}}{\partial u \partial v} \log b-16 b^{2}-\frac{1}{4} \frac{b_{u} b_{v}}{b^{2}}\right),
\end{aligned}
$$




$$
\begin{aligned}
B^{(u)}= & -\frac{1}{4}\left(2 b g-f_{v}\right)\left(\frac{\partial^{2}}{\partial u \partial v} \log b-16 b^{2}-\frac{1}{4} \frac{b_{u} b_{v}}{b^{2}}\right) \\
& -\frac{8}{3} b^{3}\left[g+\frac{9}{2} b_{u}+\frac{1}{2} \frac{b_{v v}}{b}-\frac{1}{4}\left(\frac{b_{v}}{b}\right)^{2}\right] \\
& +\frac{2}{3} b\left(f+2 b_{v}\right)\left[f+\frac{9}{2} b_{v}+\frac{1}{2} \frac{b_{u u}}{b}-\frac{1}{4}\left(\frac{b_{u}}{b}\right)^{2}\right],
\end{aligned}
$$

and $f, g$ have the values given by equations (10). Taking a linear combination of equations (11) and (16), we find that, as $u$ alone varies, the cubic (11) touches its envelope, besides at the point $y$, also at points which lie in the plane

$$
\begin{gathered}
\left\{A^{(u)}-3 \lambda-\frac{1}{6} b_{u}\left[6 b_{v}+\frac{3}{4} \frac{b_{u u}}{b}-\frac{9}{16}\left(\frac{b_{u}}{b}\right)^{2}\right]\right\} x_{3} \\
+\left(B^{(u)}+\lambda_{u}-\frac{1}{4} \frac{b_{u}}{b} \lambda\right) x_{4}=0
\end{gathered}
$$

Similarly, as $v$ alone varies, the cubic (11) touches its envelope, besides at the point $y$, also at points which lie in the plane

$$
\begin{gathered}
\left\{A^{(v)}-3 \lambda-\frac{1}{6} b_{v}\left[6 b_{u}+\frac{3}{4} \frac{b_{v v}}{b}-\frac{9}{16}\left(\frac{b_{v}}{b}\right)^{2}\right]\right\} x_{2} \\
+\left(B^{(v)}+\lambda_{v}-\frac{1}{4} \frac{b_{v}}{b}\right) x_{4}=0
\end{gathered}
$$

where $A^{(v)}$ and $B^{(v)}$ are obtained from $A^{(u)}$ and $B^{(u)}$, respectively, by interchanging $u$ and $v$. The line represented by equations (18) and (19) pierces the cubic (11) in the points where it touches its envelope, $u$ and $v$ varying.

At the close of $\S I V$ we found that for a particular value of $b$, namely $b=$ const., the surface $S$ defined by equations (14) subject to (12) is a cubic surface. We are now in a position to show that every surface defined by equations (14) and (12) is a cubic. Such a surface is a cubic in case there exists a function $\lambda$ such that the cubic (11) is independent of $u$, $v$, or is the same at every point of the surface; in this case the cubic (11) is the surface $S$ itself. Such a function $\lambda$ must be a common solution of the four equations obtained by setting equal to zero the four coefficients in equations (18) and (19). These four equations have a common solution $\lambda$, which is given by the formula 


$$
\lambda=\frac{1}{6} b_{u u}-\frac{5}{24} \frac{b_{u} b_{u u}}{b}+\frac{5}{96} b_{u}\left(\frac{b_{u}}{b}\right)^{2}+\frac{5}{3} b b_{u v}+b_{u} b_{v}
$$

(20)

$$
-\frac{1}{8} \frac{b_{v} b_{v v}}{b}+\frac{5}{96} b_{v}\left(\frac{b_{v}}{b}\right)^{2}-\frac{16}{3} b^{4} .
$$

That this function is symmetric with respect to $u$ and $v$ is easily verified by means of equations (13).

We conclude that conditions (8) are necessary and sufficient that the integrating surfaces of system (1) be cubics.

Untverestry or Chicaco,

Cnacago, Inl. 